\title{
MicroRNA-302a inhibits cell proliferation and invasion, and induces cell apoptosis in hepatocellular carcinoma by directly targeting VEGFA
}

\author{
CHENGLIN QIN, WENZHANG ZHA, RENGEN FAN, HUIMIN DING, YONGHUA XU and CHEN WANG \\ Department of General Surgery, The Fourth Affiliated Hospital of Nantong Medical College, \\ Yancheng City No. 1 People's Hospital, Yancheng, Jiangsu 224001, P.R. China
}

Received October 23, 2016; Accepted June 21, 2017

DOI: $10.3892 / \mathrm{mmr} .2017 .7312$

\begin{abstract}
Hepatocellular carcinoma (HCC) is the fifth most common cancer and the third most common cause of cancer-related mortality worldwide. An increasing number of studies have demonstrated that microRNAs may be used as diagnostic, therapeutic and prognostic targets for human cancers, including HCC. The present study aimed to evaluate microRNA (miR)-302a expression and function in HCC, and its underlying mechanisms. The results revealed that miR-302a was expressed at low levels in HCC tissues and cell lines. Reduced miR-302a expression was correlated with tumor-node-metastasis stage and lymph node metastasis in patients with HCC. Additionally, overexpression of miR-302a reduced cell proliferation and invasion, and induced apoptosis in HCC cells. Vascular endothelial growth factor A (VEGFA) was demonstrated to be a direct target gene of miR-302a. VEGFA was highly expressed in HCC tissues and inversely correlated with miR-302a expression. Knockdown of VEGFA expression led to reduced HCC cell proliferation and invasion, and increased apoptosis rates, similar to miR-302a overexpression, which suggested that VEGFA may be a functional downstream target of miR-302a in HCC. These data suggested that this newly identified miR-302a/VEGFA axis may be involved in $\mathrm{HCC}$ formation and progression. The present results also provide novel potential targets for the treatments of patients with HCC.
\end{abstract}

\section{Introduction}

Hepatocellular carcinoma (HCC) is a primary malignancy of the liver, and is the fifth most commonly diagnosed cancer and the third largest cause of cancer-related mortality

Correspondence to: Professor Wenzhang Zha, Department of General Surgery, The Fourth Affiliated Hospital of Nantong Medical College, Yancheng City No. 1 People's Hospital, 15 Yuehe Road, Yancheng, Jiangsu 224001, P.R. China

E-mail: yc_wenzhangzha@163.com

Key words: hepatocellular carcinoma, microRNA-302a, growth, invasion, apoptosis, vascular endothelial growth factor A worldwide (1). It is estimated that 39,230 new HCC cases and 27,170 mortalities may occur in 2016, based on cancer statistical data (2). A number of risk factors for HCC have been identified, including cirrhosis, hepatitis $\mathrm{B} /$ hepatitis $\mathrm{C}$ virus infection and nonalcoholic steatohepatitis (3). Currently, the main therapies for patients with HCC are liver resection, transplantation, radiofrequency ablation and adjuvant chemotherapy (4). Despite the progress in therapeutic treatments, the five-year overall survival rate for patients with HCC remains $<5 \%$ (5). In addition, $\sim 85 \%$ of patients with HCC are diagnosed with locally advanced tumor or distant metastasis, and result in poor prognosis (1). Therefore, a full understanding of the underlying mechanisms of the formation and progression of $\mathrm{HCC}$ is urgent, and investigations on novel therapeutic targets for the therapy of patients with this malignancy are required.

MicroRNAs (miRNAs) are endogenous, noncoding short RNA molecules that are 20-24 nucleotides in length (6). miRNAs function as specific gene regulators by interacting preferentially with the 3' untranslated region (3'UTR) of their target genes in a base-pairing manner, and subsequently inducing mRNA degradation or translational repression (7). To date, various miRNAs have been investigated that may serve important roles in diverse biological processes, such as cell proliferation, viability, apoptosis, cell cycle, angiogenesis, invasion, motility and differentiation (8-10). A previous study reported that $>50 \%$ of known miRNAs are located at genomic regions or in fragile sites closely correlated with cancer (11). Additional studies reported that miRNAs were abnormally expressed in a variety of human cancers, such as HCC (12), gastric cancer (13), colorectal cancer (14), glioma (15) and bladder cancer (16). These dysregulated miRNAs may act as oncogenes or tumor suppressors in tumorigenesis and tumor development, which depends on tumor type and the roles of their target genes $(17,18)$. In addition, it has been previously demonstrated that miRNAs may be used as diagnostic, therapeutic and prognostic targets for certain human cancers, including HCC (19-21).

Previous studies have shown that miR-302a was abnormally expressed and served significant roles in several human cancers (22-24). One such study revealed that miR-302a overexpression improved the radiosensitivity of radioresistant breast cancer cells to radiation therapy in vitro and in vivo (22). The upregulation of miR-302a also inhibited the cell invasion 
and metastasis of breast cancer in vitro and in vivo (23). However, little is known about the expression level and functions of miR-302a in HCC. In addition, abnormal expression of VEGFA, a 35-45 kDa heparin-binding glycoprotein and a key regulator of angiogenesis, has also been observed in multiple human cancers, including HCC $(25,26)$. Thus, the aim of the present study was to investigate miR-302a expression in HCC and its correlation with clinicopathological significance as well as VEGFA expression. In vitro functional experiments were performed to explore these roles in addition to the molecular mechanisms of action of miR-302a in HCC cell proliferation, invasion and apoptosis.

\section{Materials and methods}

Tissue samples. The present study was approved by the Ethics Committee of the Yancheng City No. 1 People's Hospital (Yancheng, China). Written informed consent was obtained from each patient enrolled in this study. A total of 47 paired $\mathrm{HCC}$ tissues and matched normal adjacent tissues (NATs) were obtained from patients with $\mathrm{HCC}$ patients that received liver resection surgery at Yancheng City No. 1 People's Hospital between January 2011 and December 2014. None of the patients received radiofrequency ablation or adjuvant chemotherapy prior to surgery. All tissues samples were immediately frozen with liquid nitrogen and subsequently stored at $-80^{\circ} \mathrm{C}$ until RNA extraction.

Cell lines and cell culture. The human HCC cell lines, HepG2 and Hep3B, were purchased from American Type Culture Collection (ATCC; Manassas, VA, USA). The human HCC cell lines, SMMC-7721 and Huh7, and the human immortalized normal liver epithelial cell line (L-O2) were purchased from Shanghai Institute of Biochemistry and Cell Biology (Shanghai, China). All cells were grown in Dulbecco's modified Eagle's medium (DMEM; Gibco; Thermo Fisher Scientific, Inc., Waltham, MA, USA) containing $10 \%$ fetal bovine serum (FBS; Gibco; Thermo Fisher Scientific, Inc.) and $1 \%$ penicillin/streptomycin, at $37^{\circ} \mathrm{C}$ in a humidified $5 \% \mathrm{CO}_{2}$.

Cell transfection. miRNA (miR)-302a mimics, miRNA negative controls (miR-NC), short interfering (si)RNA targeting vascular endothelial growth factor A (si-VEGFA) and siRNA negative control (si-NC) were purchased from Guangzhou Sagene Biotech Co. (Guangzhou, China). Prior to transfection, cells were seeded into 6-well plates in DMEM and incubated at $37^{\circ} \mathrm{C}$ until the cell density reached $60-70 \%$. Cells were transfected with miR-302a mimics (50 pmol $/ \mathrm{ml})$, $\mathrm{miR}-\mathrm{NC}(50 \mathrm{pmol} / \mathrm{ml})$, si-VEGFA $(50 \mathrm{pmol} / \mathrm{ml})$ or $\mathrm{si}-\mathrm{NC}$ (50 pmol/ml) using Lipofectamine 2000 (Invitrogen; Thermo Fisher Scientific, Inc.) at room temperature for $12 \mathrm{~h}$, following the manufacturer's protocol. Following transfection, the cell culture medium was replaced with DMEM containing $10 \%$ FBS. Following 48 h post-transfection, reverse transcription-quantitative polymerase chain reaction (RT-qPCR) was performed to detect miR-302a and VEGFA mRNA expression. The cell invasion assay and apoptosis assay were also performed at $48 \mathrm{~h}$ following transfection. Western blot analysis was conducted at $72 \mathrm{~h}$ post-transfection, and the MTT assay was performed $24 \mathrm{~h}$ following transfection.
$R T$ - $q P C R$. Total RNA was extracted from tissue $(1 \mathrm{~g})$ or cells $\left(1 \times 10^{6}\right.$ cells) using the TRIzol Reagent (Invitrogen; Thermo Fisher Scientific, Inc.), according to the manufacturer's protocol. To quantify miR-302a expression, cDNA was synthesized by reverse transcription using a TaqMan ${ }^{\circledR}$ MicroRNA Reverse Transcription kit (Applied Biosystems; Thermo Fisher Scientific, Inc.) following the manufacturer's protocol. The TaqMan microRNA assay (Applied Biosystems; Thermo Fisher Scientific, Inc.) was used to detect miR-302a expression; U6 was used as an internal control. The cycling conditions were as follows: $50^{\circ} \mathrm{C}$ for $2 \mathrm{~min}, 95^{\circ} \mathrm{C}$ for $10 \mathrm{~min}$ and then 40 cycles of denaturation at $95^{\circ} \mathrm{C}$ for $15 \mathrm{sec}$, and annealing/extension at $60^{\circ} \mathrm{C}$ for $60 \mathrm{sec}$. For VEGFA mRNA expression, total RNA was reverse transcribed to cDNA with the PrimeScript RT Reagent kit (Takara Bio, Inc., Otsu, Japan). qPCR was performed with SYBR Premix Ex Taq (Takara Bio, Inc.). $\beta$-actin was served as an internal control for VEGFA mRNA expression. The thermocycling conditions were as follows: $5 \mathrm{~min}$ at $95^{\circ} \mathrm{C}$, followed by 40 cycles of $95^{\circ} \mathrm{C}$ for $30 \mathrm{sec}$ and $65^{\circ} \mathrm{C}$ for $45 \mathrm{sec}$. The primers were designed as follows: miR-302a, 5'-CGTGGATGTACTTGCTTTGAA-3' (forward) and 5'-TCACCAAAACATGGAAGCAC-3' (reverse); U6, 5'-GCTTCGGCAGCACATATACTAAAAT-3' (forward) and 5'-CGCTTCACGAATTTGCGTGTCAT-3' (reverse); VEGFA, 5'-ACTTTCTGCTGTCTTGGGTG-3' (forward) and 5'-CTG CATGGTGATGTTGGACT-3' (reverse); and $\beta$-actin, $5^{\prime}-\mathrm{GGG}$ ACCTGACTGACTACCTC-3' (forward) and 5'-TCATACTCC TGCTTGCTGAT-3' (reverse). All reactions were performed in triplicate. The relative expression levels were calculated using the $2^{-\Delta \Delta \mathrm{Cq}}$ method (27).

MTT cell proliferation assay. The MTT assay (Sigma-Aldrich; Merck KGaA, Darmstadt, Germany) was performed to evaluate cell proliferation. Transfected cells (3,000 cells/well) were seeded into 96 -well plates $24 \mathrm{~h}$ post-transfection. Following incubation at $37^{\circ} \mathrm{C}$ for $24,36,48$, and $72 \mathrm{~h}$, MTT assay was conducted according to the manufacturer's protocol. Briefly, $20 \mu \mathrm{l}$ MTT solution $(5 \mathrm{mg} / \mathrm{ml})$ was added into each well and incubated for $4 \mathrm{~h}$ at $37^{\circ} \mathrm{C}$. The medium was then replaced with $150 \mu \mathrm{l}$ DMSO (Sigma-Aldrich; Merck KGaA) to dissolve the purple formazan crystals, and absorbance at $490 \mathrm{~nm}$ was determined using a Tecan Infinite M200 microplate reader (Tecan Group Ltd., Männedorf, Switzerland). This assay was repeated three times.

Cell invasion assay. The invasive ability of cells was assessed using a Corning Costar Transwell Chamber (Corning, Inc., Corning, NY, USA) coated with Matrigel (BD Biosciences, San Jose, CA, USA). Transfected cells $\left(5 \times 10^{4}\right)$ in FBS-free DMEM were seeded into the upper chamber at $48 \mathrm{~h}$ post-transfection. DMEM (500 $\mu \mathrm{l})$ supplemented with $20 \%$ FBS was added into the lower chamber. Following incubation at $37^{\circ} \mathrm{C}$ for $48 \mathrm{~h}$, cells that remained on top surface of the membrane were removed with cotton swabs. Cells on the bottom surface of the membrane were fixed with $100 \%$ methanol for $10 \mathrm{~min}$ and stained with $0.5 \%$ crystal violet for $10 \mathrm{~min}$ at room temperature. A total of five randomly selected fields of the invading cells were captured and counted with an inverted microscope (Olympus Corporation, Tokyo, Japan). This assay was repeated three times. 
A

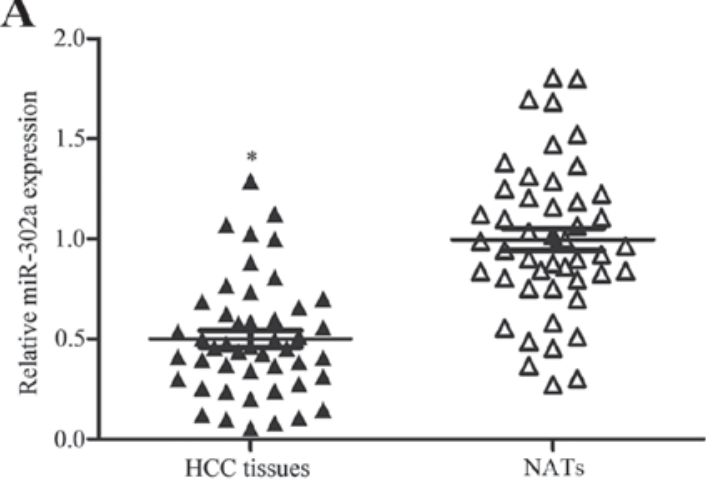

B

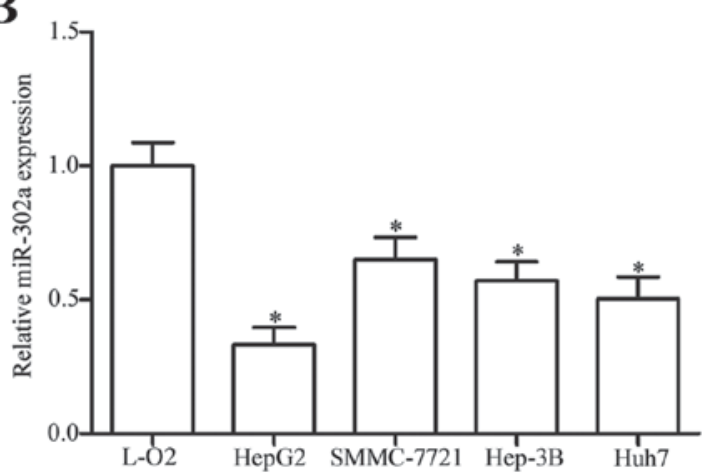

Figure 1. miR-302a expression in HCC tissues and HCC cell lines. Reverse transcription-quantitative polymerase chain reaction was uses to analyze the relative levels of miR-302a expression in (A) HCC tissues and matched NATs, and (B) HCC cell lines (HepG2, SMMC-7721, Hep3B and Huh7) and a human immortalized normal liver epithelial cell (L-O2). "P<0.05 vs. NAT or L-O2. HCC, hepatocellular carcinoma; NAT, normal adjacent tissue.

Apoptosis assay. Apoptotic rates were determined using the fluorescein isothiocyanate (FITC)-Annexin V Apoptosis Detection kit I (BD Biosciences), according to the manufacturer's protocol. Following $48 \mathrm{~h}$ transfection, cells in 6 -well plates $\left(1 \times 10^{6}\right.$ cells/well) were harvested using $0.25 \%$ trypsin (Gibco; Thermo Fisher Scientific, Inc.), washed twice with cold PBS, centrifuged at $500 \mathrm{xg}$ for $5 \mathrm{~min}$ at $4^{\circ} \mathrm{C}$, and resuspended in $1 \mathrm{X}$ binding buffer. Subsequently, cells were transferred to a $5 \mathrm{ml}$ culture tube, and $5 \mu$ l FITC-annexin $\mathrm{V}$ and $10 \mu \mathrm{l}$ propidium iodide (PI) were added into the culture tube. Following incubation at room temperature in the dark for $15 \mathrm{~min}$, apoptosis was analyzed using a flow cytometer (Beckman Coulter, Inc., Brea, CA, USA) within $1 \mathrm{~h}$ of staining. Data were analyzed using CellQuest ${ }^{\circledR}$ software (version 3.3; BD Biosciences) and experiments repeated three times.

miRNA target prediction. Potential targets of miR-302a were predicted using the algorithms provided in the online target prediction sites TargetScan (http://www.targetscan.org) and PicTar (http://pictar.mdc-berlin.de).

Dual-luciferase reporter assay. Wild-type and mutant 3'UTRs of VEGFA were synthesized by Shanghai GenePharma Co., Ltd. (Shanghai, China) and also subcloned into the pmirGLO vector; they were subsequently named pmirGLO-Wt-VEGFA-3'UTR and pmirGLO-Mut-VEGFA-3'UTR, respectively. For the luciferase reporter assay, HEK293T cells (ATCC) were seeded into the 24 -well plates at a density of $50-60 \%$ confluence. Following overnight incubation at $37^{\circ} \mathrm{C}$, cells were transfected with either pmirGLO-Wt-VEGFA-3'UTR or pmirGLO-Mut-VEGFA-3'UTR, and either miR-302a mimics or miR-NC, at room temperature using Lipofectamine 2000. Firefly and Renilla luciferase activities were detected at $48 \mathrm{~h}$ post-transfection with Dual-Luciferase Reporter Assay System (Promega Corporation, Madison, WI, USA). The relative luciferase activity was normalized to Renilla luciferase activity and experiments were repeated three times.

Western blot analysis. Cell proteins were extracted using radioimmunoprecipitation assay buffer containing a protease inhibitor cocktail (Sigma-Aldrich; Merck KGaA). The protein concentrations were quantified using a Bicinchoninic Acid assay kit (Pierce; Thermo Fisher Scientific, Inc.). Equal proteins $(20 \mu \mathrm{g})$ were separated by $10 \%$ SDS-PAGE and subsequently transferred to polyvinylidene difluoride membranes (EMD Millipore, Billerica, MA, USA). Membranes were blocked with 5\% skimmed in Tris-buffered saline containing $0.1 \%$ Tween-20 (TBST) at room temperature for $1 \mathrm{~h}$, followed by incubation with primary antibodies against VEGFA (1:1,000 dilution; cat. no. sc-65617) and GADPH (1:1,000 dilution; cat. no. sc-32233; Santa Cruz Biotechnology, Inc., Dallas, TX, USA) at $4{ }^{\circ} \mathrm{C}$ overnight. Subsequently, the membranes were washed three times with TBST and incubated with horseradish peroxidase-conjugated goat anti-mouse secondary antibodies $(1: 5,000$ dilution; cat. no. sc-2005; Santa Cruz Biotechnology, Inc.) at room temperature for $1 \mathrm{~h}$. Protein bands were visualized using Chemiluminescence Detection Reagents (Pierce; Thermo Fisher Scientific, Inc.). GADPH was used as a loading control and experiments were repeated three times.

Statistical analysis. Data are expressed as the mean \pm standard deviation and calculated with SPSS version 19.0 (IBM Corp., Armonk, NY, USA). Correlations between mRNA and miRNA expression were determined by Spearman's correlation analysis. Student's t-tests and Pearson's $\chi^{2}$ test were used to compare the differences between two groups. $\mathrm{P}<0.05$ was considered to indicate a statistically significant difference.

\section{Results}

miR-302a is underexpressed in HCC tissues and cell lines. To understand the biological roles of miR-302a in HCC development, the expression levels were examined in HCC tissues and compared with matched NATs. RT-qPCR analysis revealed that miR-302a expression was significantly reduced in HCC tissues compared with expression in the matched NATs (Fig. 1A; P $<0.05$ ). Similarly, miR-302a expression levels were lower in the HCC cell lines HepG2, SMMC-7721, Hep3B and Huh7 compared with the level of expression in the L-O2 human immortalized normal liver epithelial cell (Fig. 1B; $\mathrm{P}<0.05$ ). These results suggested that 
Table I. Correlations between clinicopathological characteristics and miR-302a expression in patients with hepatocellular carcinoma.

miR-302a expression

\begin{tabular}{|c|c|c|c|c|}
\hline \multirow[b]{2}{*}{ Clinicopathological significances } & \multirow[b]{2}{*}{ Cases (n) } & & \multirow[b]{2}{*}{ P-value } \\
\hline & & Low & High & \\
\hline Age (years) & & & & 0.510 \\
\hline$<50$ & 25 & 16 & 9 & \\
\hline$\geq 50$ & 22 & 12 & 10 & \\
\hline Sex & & & & 0.919 \\
\hline Male & 35 & 21 & 14 & \\
\hline Female & 12 & 7 & 5 & \\
\hline Tumor size $(\mathrm{cm})$ & & & & 0.440 \\
\hline$<5$ & 24 & 13 & 11 & \\
\hline$\geq 5$ & 23 & 15 & 8 & \\
\hline TNM stage & & & & 0.028 \\
\hline I-II & 23 & 10 & 13 & \\
\hline III-IV & 24 & 18 & 6 & \\
\hline Differentiation & & & & 0.595 \\
\hline Well/moderate & 25 & 14 & 11 & \\
\hline Poor/undifferentiated & 22 & 14 & 8 & \\
\hline Lymph node metastasis & & & & 0.037 \\
\hline Negative & 26 & 12 & 14 & \\
\hline Positive & 21 & 16 & 5 & \\
\hline
\end{tabular}

miR, microRNA; TNM, tumor-node-metastasis.

miR-302a may serve important roles in HCC formation and progression.

Association between miR-302a expression and clinicopathological significances of patients with HCC. The clinicopathological significance of miR-302a expression in patients with HCC was also investigated (Table I). Low miR-302a expression was significantly correlated with TNM stage $(\mathrm{P}=0.028)$ and lymph node metastasis $(\mathrm{P}=0.037)$, but not with age $(\mathrm{P}=0.510)$, sex $(\mathrm{P}=0.919)$, differentiation $(\mathrm{P}=0.595)$ or tumor size $(\mathrm{P}=0.440)$.

miR-302a attenuates cell proliferation, invasion and induces apoptosis in HCC. To investigate the roles of miR-302a in HCC progression, HepG2 and Huh7 cells were transfected with miR-302a mimics to increase endogenous miR-302a expression level, which was confirmed by RT-qPCR analysis (Fig. 2A; $\mathrm{P}<0.05$ vs. miR-NC). The effects of miR-302a expression on HCC cell proliferation, invasion and apoptosis were also examined. MTT assay results demonstrated that miR-302a overexpression inhibited the proliferation of HepG2 and Huh7 cells compared with control miR-NC transfected cells (Fig. 2B and C, respectively; $\mathrm{P}<0.05$ at 48 and $72 \mathrm{~h}$ ). Similarly, cell invasion assays revealed that the number of invading HepG2 and Huh7 cells was significantly reduced following transfection with miR-302a mimics compared with cells transfected with miR-NC (Fig. 2D; P<0.05). In addition, overexpression of miR-302a significantly enhanced apoptotic rates in HepG2 and Huh7 cells (Fig. 2E; $\mathrm{P}<0.05$ vs. miR-NC). These results suggested that miR-302a may serve an important role in the occurrence and progression of HCC.

miR-302a reduces VEGFA expression by directly binding to its 3'UTR. miR-302a was demonstrated to act as a tumor suppressor in HCC; therefore, the mechanisms underlying the tumor suppressive roles of miR-302a in HCC were examined. Candidate targets of miR-302a were queried using online bioinformatics databases; the results indicated that the 3'UTR of VEGFA contained complementary sites for miR-302a (Fig. 3A). To verify this hypothesis, RT-qPCR and western blotting were used to examine the endogenous levels of VEGFA mRNA and protein expression in HepG2 and Huh7 cells transfected with miR-302a mimics or miR-NC. Overexpression of miR-302a significantly reduced both the mRNA (Fig. 3B; $\mathrm{P}<0.05$ ) and the protein (Fig. 3C; $\mathrm{P}<0.05)$ level of VEGFA expression in HepG2 and Huh7 cells compared with cells transfected with miR-NC. In addition, the dual-luciferase reporter assay was performed to explore whether miR-302a was able to directly target the 3'UTR of VEGFA. The results demonstrated that miR-302a overexpression led to decreased luciferase activity in HEK293T cells co-transfected with pmirGLO-Wt-VEGFA-3'UTR (Fig. 3D; $\mathrm{P}<0.05$ vs. miR-NC). However, no significant differences were identified in the luciferase activity of cells co-transfected with miR-302a mimics and pmirGLO-Mut-VEGFA-3'UTR 
A

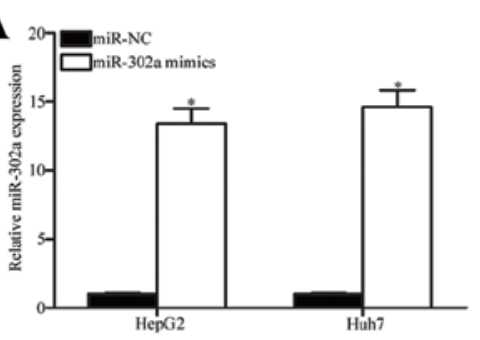

B

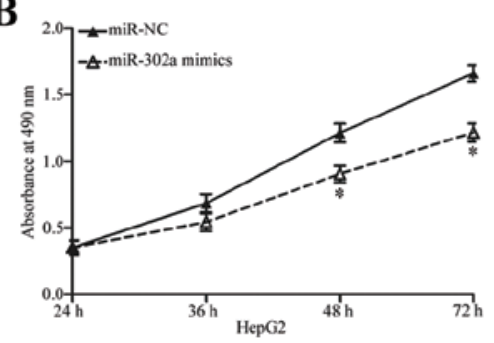

C

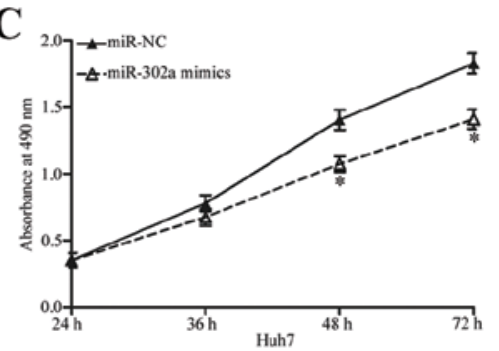

D
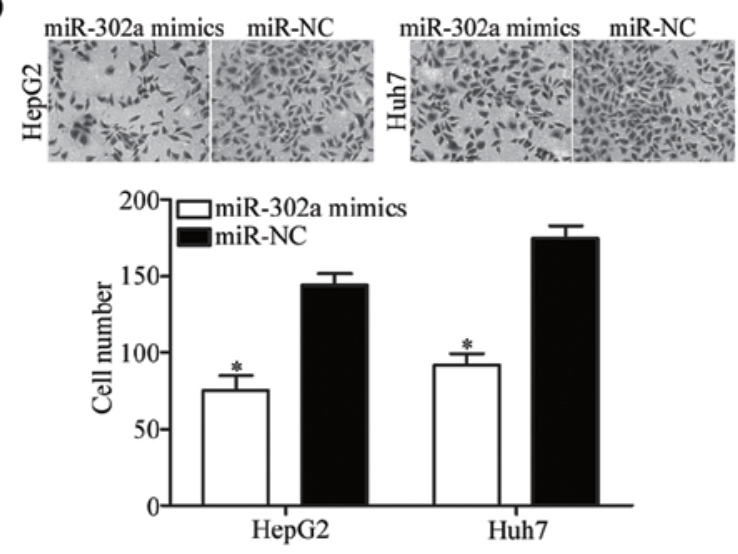

$\mathbf{E}$
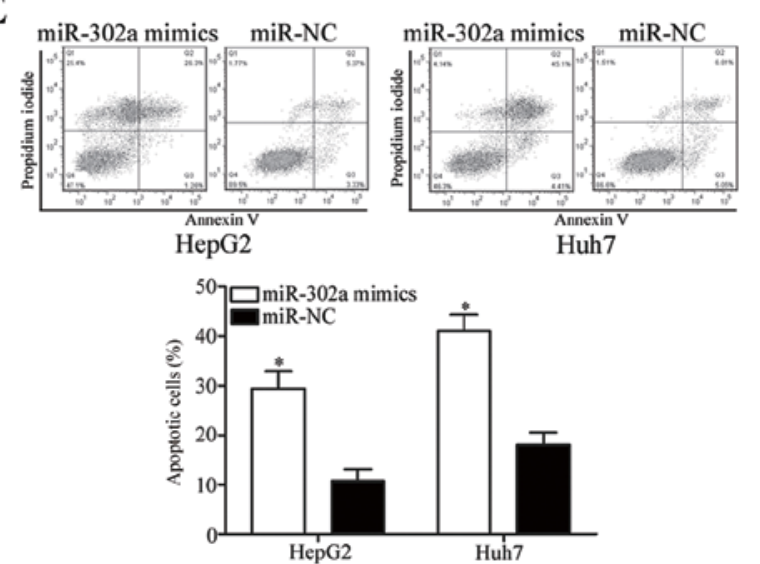

Figure 2. miR-302a inhibits proliferation and invasion, and induces apoptosis in HepG2 and Huh7 hepatocellular carcinoma cells. (A) Reverse transcription-quantitative polymerase chain reaction was performed to examine miR-302a expression in HepG2 and Huh7 cells transfected with miR-302a mimics or miR-NC. The MTT assay was used to evaluate cell proliferation in (B) HepG2 and (C) Huh7 cells transfected with miR-302a mimics or miR-NC. (D) Cell invasion assays were performed to determine the invasive ability of HepG2 and Huh7 cells following transfection with miR-302a mimics or miR-NC (magnification, x200). (E) Apoptosis assays were carried out using flow cytometry to determine apoptotic rates in HepG2 and Huh7 cells following transfection with miR-302a mimics or miR-NC. ${ }^{*} \mathrm{P}<0.05$ vs. miR-NC. miR, microRNA; NC, negative control.

A

$$
\begin{aligned}
\text { Wt-VEGFA-3'UTR } & 5 \text {,...GGAGACUCUGCGCAGAGCACUUU... } \\
\text { hsa-miR-302a } & 3 \text { ' } 1111 \text { AGUGGUUUUGUACCUUCGUGAAU }
\end{aligned}
$$

Mut-VEGFA-3'UTR 5'...GGAGACUCUGCGCAGUCGUGAAU...

\section{B}

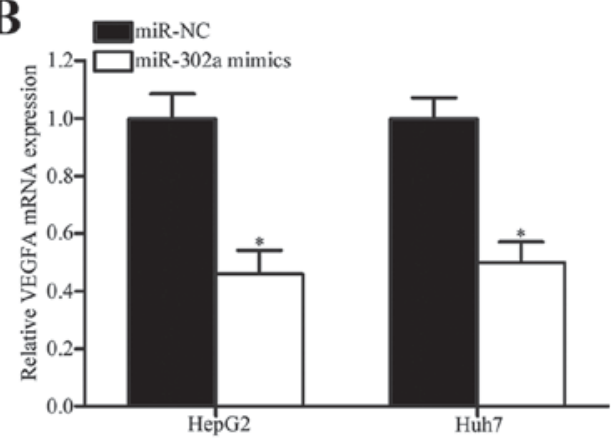

D

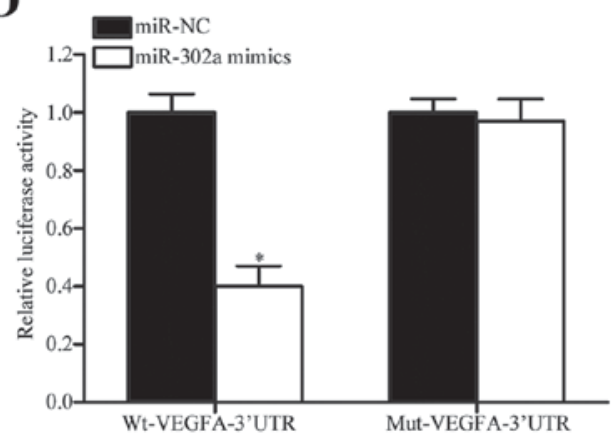

Figure 3. VEGFA is a direct target of miR-302a. (A) Predicted binding sites of miR-302a in the wild-type and mutant 3'UTR of VEGFA. (B) Reverse transcription-quantitative polymerase chain reaction analysis of VEGFA mRNA expression in HepG2 and Huh7 cells transfected with miR-302a mimics or miR-NC. (C) Expression levels of VEGFA protein in HepG2 and Huh7 cells transfected with miR-302a mimics or miR-NC were detected by western blotting. (D) Dual-luciferase reporter assays were performed in HEK293T cells co-transfected with miR-302a mimics or miR-NC, and pmirGLO-Wt-VEGFA-3'UTR or pmirGLO-Mut-VEGFA-3'UTR. "P<0.05 vs. miR-NC. miR, microRNA; mut, mutant; NC, negative control; UTR, untranslated region; VEGFA, vascular endothelial growth factor A; wt, wild type. 
A

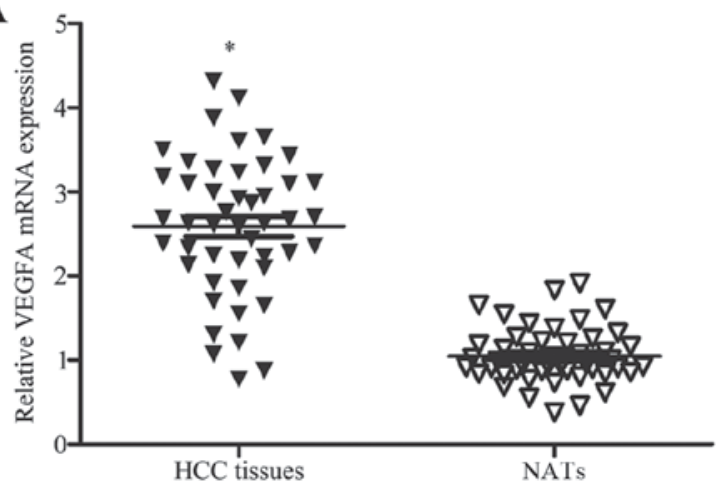

B

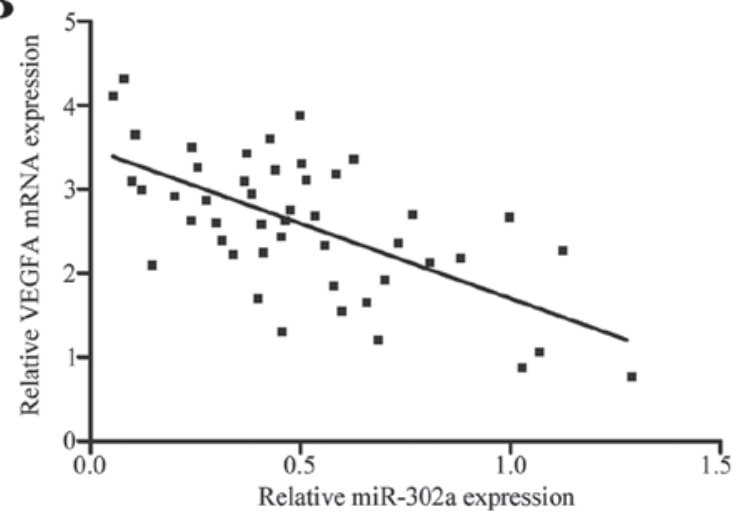

Figure 4. VEGFA is inversely correlated with miR-302a expression in HCC tissues. (A) Reverse transcription-quantitative polymerase chain reaction was used to determine the levels of VEGFA mRNA expression in HCC tissues compared with matched NATs. "P<0.05 vs. NATs. (B) The correlation between VEGFA mRNA and miR-302a expression was analyzed in HCC tissues using Spearman's correlation analysis. HCC, hepatocellular carcinoma; miR, microRNA; NAT, normal adjacent tissue; VEGFA, vascular endothelial growth factor A.
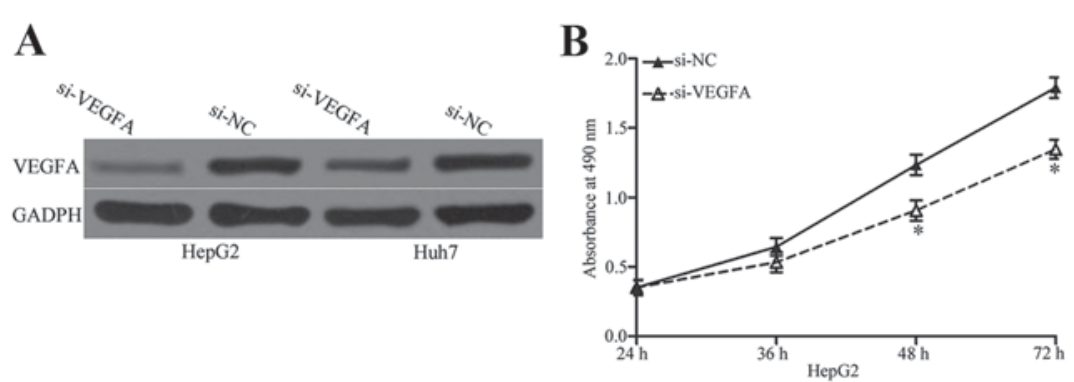

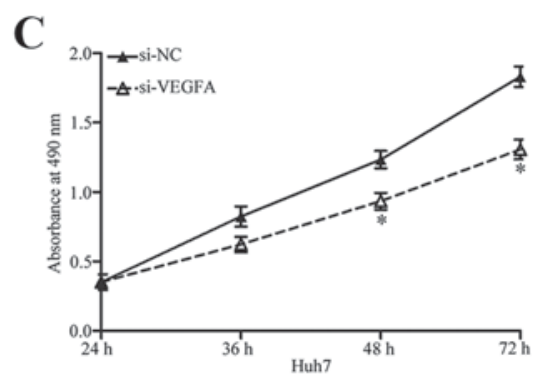

E

D
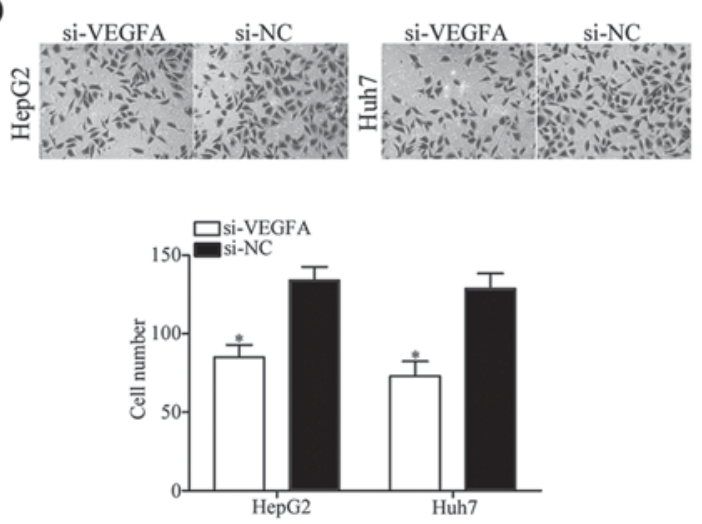
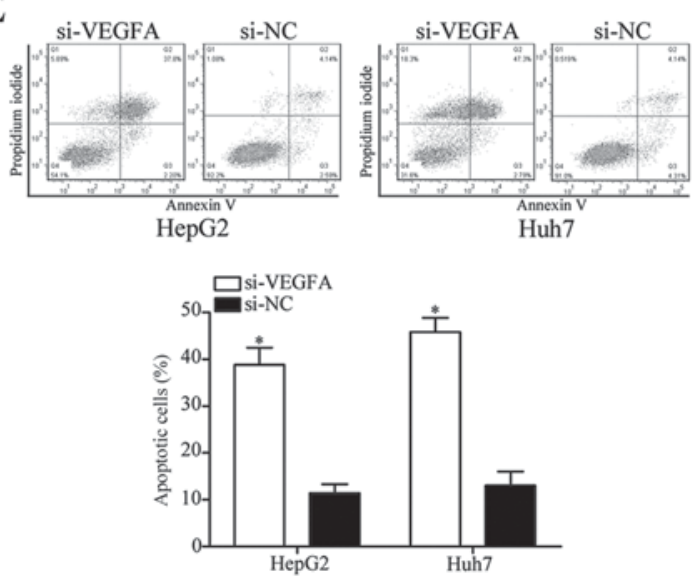

Figure 5. VEGFA underexpression suppresses cell proliferation and invasion, and enhances apoptosis in hepatocellular carcinoma cells. (A) Western blot analysis demonstrated that si-VEGFA effectively decreased VEGFA expression in HepG2 and Huh7 cells. Decreased expression of VEGFA in (B) HepG2 and (C) Huh7 cells reduced cell proliferation. (D) VEGFA knockdown decreased the invasive capacity in HepG2 and Huh7 cells (magnification, x200). (E) Inhibition of VEGFA increased the rate of apoptosis in HepG2 and Huh7 cells. "P<0.05 vs. si-NC. HCC, hepatocellular carcinoma; si, small interfering RNA; VEGFA, vascular endothelial growth factor A.

compared with miR-NC. These results suggested that miR-302a may target the 3'UTR of VEGFA and reduce its expression in HCC.

VEGFA expression is negatively correlated with miR-302a expression in HCC tissues. VEGFA has previously been reported to be upregulated in HCC $(25,26)$. As VEGFA has been identified as a direct target gene of miR-302a, the present study hypothesized that reduced expression of miR-302a may be involved in the high mRNA expression level of VEGFA in HCC. To confirm this hypothesis, RT-qPCR was conducted to measure VEGFA mRNA expression in HCC tissues and NATs. VEGFA mRNA expression was significantly higher in HCC tissues compared with expression in NATs (Fig. 4A; $\mathrm{P}<0.05)$. In addition, the correlation between miR-302a and VEGFA expression in HCC tissues was analyzed using Spearman's correlation analysis. The results indicated that VEGFA mRNA expression levels were negatively correlated 
with miR-302a expression in HCC tissues (Fig. 4B; $r=-0.6261$; $\mathrm{P}<0.001)$. These results further demonstrated that VEGFA may be a direct target of miR-302a, and that decreased miR302a expression may contribute to the upregulation of VEGFA in HCC.

miR-302a inhibits cell proliferation and invasion, and induces apoptosis in HCC by targeting VEGFA. To determine whether VEGFA expression was involved in the tumor suppressive roles induced by miR-302a overexpression in $\mathrm{HCC}$, its biological roles in HCC progression were evaluated. si-VEGFA was used to knockdown VEGFA expression in HepG2 and Huh7 cells (Fig. 5A). MTT assay, cell invasion assay and apoptosis assay results revealed that knockdown of VEGFA significantly inhibited cell proliferation (Fig. 5B and C; $\mathrm{P}<0.05$ at 48 and $72 \mathrm{~h}$ ) and invasion (Fig. 5D; $\mathrm{P}<0.05$ ), and significantly induced apoptosis (Fig. 5E; $\mathrm{P}<0.05$ ) in HepG2 and Huh7 cells compared with cells transfected with si-NC. The results induced by VEGFA knockdown in HCC cell proliferation, invasion and apoptosis were similar to those in cells overexpressing miR-302a, which suggested that miR-302a may act as a tumor suppressor in HCC progression by targeting the expression of VEGFA.

\section{Discussion}

A number of previous studies have indicated that abnormally expressed miRNAs may be involved in the occurrence and progression of HCC by the negative regulation of their target genes $(28,29)$. Therefore, further investigation of potential miRNAs that may contribute to the initiation and progression of HCC may provide novel targets for the diagnosis and treatments of patients with this malignancy (30). In the present study miR-302a was demonstrated to be significantly downregulated both in HCC tissues and in HCC cell lines. Low miR-302a expression was significantly correlated with TNM stage and lymph node metastasis in patients with HCC. miR-302a overexpression reduced cell proliferation and invasion, and induced apoptosis in HCC cells. In addition, VEGFA was validated as a direct target gene of miR-302a in HCC cells. These findings suggested that miR-302a underexpression may serve important roles in HCC formation and progression.

miR-302a has been previously reported to act as a tumor suppressor in different cancer types (22-24). For example, miR-302a expression was low in irradiated breast cancer cells. Upregulation of miR-302a expression enhanced radiosensitivity of radioresistant breast cancer cells to radiation therapy in vitro and in vivo (22). Another study reported that expression levels of miR-302a were decreased in metastatic breast cancer cells and tumor tissues (23). Overexpression of miR-302a suppressed breast cancer cell invasion and metastasis in vitro and in vivo (23). In testicular embryonal carcinoma, miR-302a overexpression was demonstrated to improve the sensitivity of NT2 cells to cisplatin by inducing cell apoptosis (24). In ovarian cancer, miR-302a was revealed to be downregulated in tumor tissues and cell lines (31). Low miR-302a expression was correlated with tumor stage of ovarian cancer. Ectopic expression of miR-302a inhibited ovarian cancer cell proliferation and enhanced the cell cycle progress. In prostate cancer, miR-302a expression was reported to be lower in tumor tissues and cell lines (32). Expression levels of miR-302a were significantly associated with Gleason score. Upregulation of miR-302a in prostate cancer cells enhanced G1/S cell cycle arrest and repressed cell proliferation in vitro and in vivo. A previous study demonstrated that miR-302a was reduced in colorectal cancer cells (33). Resumption expression of miR-302a decreased cell proliferation and invasion in colorectal cancer. These findings suggested that miR-302a may be a novel therapeutic strategy for patients with these cancer types.

miRNAs participate in various physiological or pathological biological processes by negatively regulating the expression of their target genes; therefore, it is essential to identify the direct target genes of miR-302a in HCC. Previous studies have indicated that miR-302a targets many important genes, such as AKT serine/threonine kinase 1 (AKT1), RAD52 homolog DNA repair protein and C-X-C motif chemokine receptor $4(22,23)$ in breast cancer, syndecan 1 in ovarian cancer (31), AKT in prostate cancer (32), and mitogen-activated protein kinase in colorectal cancer (33). The present study used bioinformatics analysis and the luciferase reporter assay to demonstrate that the 3'UTR of VEGFA is a direct target gene of miR-302a in HCC. In addition, miR-302a was demonstrated to negatively regulate VEGFA expression at both the mRNA and protein level in HCC. VEGFA mRNA was significantly expressed in HCC tissues and was inversely correlated with miR-302a expression. In addition, similar effects were observed when VEGFA expression was knocked down in HCC as when miR-302a was overexpressed.

VEGFA is a $35-45 \mathrm{kDa}$ heparin-binding glycoprotein and a key regulator of angiogenesis (34); angiogenesis is known to be a crucial factor in local tumor growth tumors and metastasis progression. An increasing number of studies have indicated that VEGFA was abnormally expressed in multiple human cancers, including HCC $(25,26)$, gastric cancer (35), bladder cancer (36), retinoblastoma (37) and colorectal cancer (38). Additional studies have demonstrated that VEGFA serves important roles in vasculogenesis, angiogenesis, cell proliferation, migration, invasion, apoptosis and tumor angiogenesis (39-42). Therefore, targeting VEGFA may be an effective therapeutic strategy for patients with certain tumors. Several agents that target VEGFA are in development, some of which are in clinical trials for cancer treatment (34). The present study demonstrated that miR-302a targeted VEGFA to inhibit HCC cell growth and metastasis, and enhanced apoptosis. These results suggested that the miR-302a/VEGFA pathway may provide novel therapeutic targets for patients with HCC.

In conclusion, miR-302a may act as a tumor suppressor in HCC, at least in part, via the inhibition of VEGFA expression, which suggested that the miR-302a/VEGFA axis may provide novel therapeutic targets for the treatment of HCC. Future studies should investigate the upstream regulatory mechanism underlying miR-302a expression.

\section{References}

1. El-Serag HB and Rudolph KL: Hepatocellular carcinoma: Epidemiology and molecular carcinogenesis. Gastroenterology 132: 2557-2576, 2007.

2. Siegel RL, Miller KD and Jemal A: Cancer statistics, 2016. CA Cancer J Clin 66: 7-30, 2016. 
3. Dhanasekaran R, Limaye A and Cabrera R: Hepatocellular carcinoma: Current trends in worldwide epidemiology, risk factors, diagnosism, and therapeutics. Hepat Med 4: 19-37, 2012.

4. Xu L, Zhang M, Zheng X, Yi P, Lan C and Xu M: The circular RNA ciRS-7 (Cdrlas) acts as a risk factor of hepatic microvascular invasion in hepatocellular carcinoma. J Cancer Res Clin Oncol 143: 17-27, 2017.

5. Hou B, Jian Z, Chen S, Ou Y, Li S and Ou J: Expression of miR-216a in pancreatic cancer and its clinical significance. Nan Fang Yi Ke Da Xue Xue Bao 32: 1628-1631, 2012. (In Chinese)

6. Bartel DP: MicroRNAs: Genomics, biogenesis, mechanism, and function. Cell 116: 281-297, 2004.

7. Krol J, Loedige I and Filipowicz W: The widespread regulation of microRNA biogenesis, function and decay. Nat Rev Genet 11 597-610, 2010

8. Choi E, Choi E and Hwang KC: MicroRNAs as novel regulators of stem cell fate. World J Stem Cells 5: 172-187, 2013.

9. Zhou W, Zou B, Liu L, Cui K, Gao J, Yuan S and Cong N: MicroRNA-98 acts as a tumor suppressor in hepatocellular carcinoma via targeting SALL4. Oncotarget 7: 74059-74073, 2016.

10. Wang CY, Zhang JJ, Hua L, Yao KH, Chen JT and Ren XQ MicroRNA-98 suppresses cell proliferation, migration and invasion by targeting collagen triple helix repeat containing 1 in hepatocellular carcinoma. Mol Med Rep 13: 2639-2644, 2016

11. Calin GA, Sevignani C, Dumitru CD, Hyslop T, Noch E, Yendamuri S, Shimizu M, Rattan S, Bullrich F, Negrini M and Croce CM: Human microRNA genes are frequently located at fragile sites and genomic regions involved in cancers. Proc Natl Acad Sci USA 101: 2999-3004, 2004

12. Liu S, Liu K, Zhang W, Wang Y, Jin Z, Jia B and Liu Y: miR-449a inhibits proliferation and invasion by regulating ADAM10 in hepatocellular carcinoma. Am J Transl Res 8: 2609-2619, 2016.

13. Wang LL, Wang L, Wang XY, Shang D, Yin SJ, Sun LL and Ji HB: MicroRNA-218 inhibits the proliferation, migration, and invasion and promotes apoptosis of gastric cancer cells by targeting LASP1. Tumour Biol 37: 15241-15252, 2016.

14. Li H, Zhang H, Lu G, Li Q, Gu J, Song Y, Gao S and Ding Y: Mechanism analysis of colorectal cancer according to the microRNA expression profile. Oncol Lett 12: 2329-2336, 2016.

15. Peng G, Liao Y and Shen C: miRNA-429 inhibits astrocytoma proliferation and invasion by targeting BMI1. Pathol Oncol Res 23: 369-376, 2017

16. Wu D, Niu X, Pan H, Zhou Y, Qu P and Zhou J: MicroRNA-335 is downregulated in bladder cancer and inhibits cell growth, migration and invasion via targeting ROCK1. Mol Med Rep 13: 4379-4385, 2016

17. Wang Q, Huang Z, Guo W, Ni S, Xiao X, Wang L, Huang D, Tan C, Xu Q, Zha R, et al: microRNA-202-3p inhibits cell proliferation by targeting ADP-ribosylation factor-like 5A in human colorectal carcinoma. Clin Cancer Res 20: 1146-1157, 2014.

18. Josson S, Gururajan M, Hu P, Shao C, Chu GY, Zhau HE, Liu C, Lao K, Lu CL, Lu YT, et al: miR-409-3p/-5p promotes tumorigenesis, epithelial-to-mesenchymal transition, and bone metastasis of human prostate cancer. Clin Cancer Res 20: 4636-4646, 2014

19. Fan MQ, Huang CB, Gu Y, Xiao Y, Sheng JX and Zhong L: Decrease expression of microRNA-20a promotes cancer cell proliferation and predicts poor survival of hepatocellular carcinoma. J Exp Clin Cancer Res 32: 21, 2013

20. Du C, Weng X, Hu W, Lv Z, Xiao H, Ding C, Gyabaah OA, Xie H, Zhou L, Wu J and Zheng S: Hypoxia-inducible MiR-182 promotes angiogenesis by targeting RASA1 in hepatocellular carcinoma. J Exp Clin Cancer Res 34: 67, 2015.

21. Chuang KH, Whitney-Miller CL, Chu CY, Zhou Z, Dokus MK, Schmit S and Barry CT: MicroRNA-494 is a master epigenetic regulator of multiple invasion-suppressor microRNAs by targeting ten eleven translocation 1 in invasive human hepatocellular carcinoma tumors. Hepatology 62: 466-480, 2015

22. Liang Z, Ahn J, Guo D, Votaw JR and Shim H: MicroRNA-302 replacement therapy sensitizes breast cancer cells to ionizing radiation. Pharm Res 30: 1008-1016, 2013.

23. Liang Z, Bian X and Shim H: Inhibition of breast cancer metastasis with microRNA-302a by downregulation of CXCR4 expression. Breast Cancer Res Treat 146: 535-542, 2014.
24. Liu L, Lian J, Zhang H, Tian H, Liang M, Yin M and Sun F: MicroRNA-302a sensitizes testicular embryonal carcinoma cells to cisplatin-induced cell death. J Cell Physiol 228: 2294-2304, 2013.

25. Yamaguchi R, Yano H, Iemura A, Ogasawara S, Haramaki M and Kojiro M: Expression of vascular endothelial growth factor in human hepatocellular carcinoma. Hepatology 28: 68-77, 1998.

26. Miura H, Miyazaki T, Kuroda M, Oka T, Machinami R, Kodama T, Shibuya M, Makuuchi M, Yazaki Y and Ohnishi S: Increased expression of vascular endothelial growth factor in human hepatocellular carcinoma. J Hepatol 27: 854-861, 1997.

27. Livak KJ and Schmittgen TD: Analysis of relative gene expression data using real-time quantitative PCR and the 2(-Delta Delta C(T)) method. Methods 25: 402-408, 2001

28. Mao B and Wang G: MicroRNAs involved with hepatocellular carcinoma (Review). Oncol Rep 34: 2811-2820, 2015.

29. Giordano $\mathrm{S}$ and Columbano A: MicroRNAs: New tools for diagnosis, prognosis, and therapy in hepatocellular carcinoma? Hepatology 57: 840-847, 2013.

30. Jin H, Yu M, Lin Y, Hou B, Wu Z, Li Z and Sun J: MiR-502-3P suppresses cell proliferation, migration, and invasion in hepatocellular carcinoma by targeting SET. Onco Targets Ther 9: 3281-3289, 2016

31. Guo T, Yu W, Lv S, Zhang C and Tian Y: MiR-302a inhibits the tumorigenicity of ovarian cancer cells by suppression of SDC1. Int J Clin Exp Pathol 8: 4869-4880, 2015.

32. Zhang GM, Bao CY, Wan FN, Cao DL, Qin XJ, Zhang HL, Zhu Y, Dai B, Shi GH and Ye DW: MicroRNA-302a suppresses tumor cell proliferation by inhibiting AKT in prostate cancer. PLoS One 10: e0124410, 2015.

33. Wei ZJ, Tao ML, Zhang W, Han GD, Zhu ZC, Miao ZG, Li JY and Qiao ZB: Up-regulation of microRNA-302a inhibited the proliferation and invasion of colorectal cancer cells by regulation of the MAPK and PI3K/Akt signaling pathways. Int J Clin Exp Pathol 8: 4481-4491, 2015.

34. Fan L, Wu Q, Xing X, Wei Y and Shao Z: MicroRNA-145 targets vascular endothelial growth factor and inhibits invasion and metastasis of osteosarcoma cells. Acta Biochim Biophys Sin (Shanghai) 44: 407-414, 2012

35. Ji YN, Wang Q, Li Y and Wang Z: Prognostic value of vascular endothelial growth factor A expression in gastric cancer: A meta-analysis. Tumour Biol 35: 2787-2793, 2014.

36. Huang YJ, Qi WX, He AN, Sun YJ, Shen Z and Yao Y: Prognostic value of tissue vascular endothelial growth factor expression in bladder cancer: A meta-analysis. Asian Pac J Cancer Prev 14: 645-649, 2013

37. Youssef NS and Said AM: Immunohistochemical expression of CD117 and vascular endothelial growth factor in retinoblastoma: Possible targets of new therapies. Int J Clin Exp Pathol 7: 5725-5737, 2014

38. Wen L, Wang R, Lu X and You C: Expression and clinical significance of vascular endothelial growth factor and fms-related tyrosine kinase 1 in colorectal cancer. Oncol Lett 9: 2414-2418, 2015.

39. Zhuang $\mathrm{Y}$ and Wei M: Impact of vascular endothelial growth factor expression on overall survival in patients with osteosarcoma: A meta-analysis. Tumour Biol 35: 1745-1749, 2014.

40. Liu Y, Zheng Q, Wu H, Guo X, Li J and Hao S: Rapamycin increases pCREB, Bcl-2, and VEGF-A through ERK under normoxia. Acta Biochim Biophys Sin (Shanghai) 45: 259-267, 2013.

41. Wiszniak S, Mackenzie FE, Anderson P, Kabbara S, Ruhrberg C and Schwarz Q: Neural crest cell-derived VEGF promotes embryonic jaw extension. Proc Natl Acad Sci USA 112: 6086-6091, 2015

42. Wu YY, Chen L, Wang GL, Zhang YX, Zhou JM, He S, Qin J and Zhu YY: Inhibition of hepatocellular carcinoma growth and angiogenesis by dual silencing of NET-1 and VEGF. J Mol Histol 44: 433-445, 2013 